\title{
ON CONFORMALLY COMPACT EINSTEIN MANIFOLDS
}

\author{
XIAODONG WANG
}

\section{Introduction}

One of the new ideas under investigation in string theory is ADS/CFT correspondence. It has a beautiful mathematical formulation and provides new stimuli and insights to the study of conformally compact Einstein manifolds. By the work of Lee [14] and recent results of Witten-Yau [22] we expect that there should be a very interesting relationship between the geometry of a conformally compact Einstein manifold and the conformal geometry of its conformal infinity.

In this paper we establish several results which support this philosophy. A common theme in the approach is Bochner technique. In Section 3 we prove the following theorem.

Theorem 1.1. Let $(M, g)$ be a conformally compact Riemannian manifold of dimension $n+1$ with Ric $\geq-n$.

1. If $\lambda_{0}(g)>n-1$, then $H_{n}(M, \mathbb{Z})=0$. In particular, the conformal infinity is connected.

2. If $\lambda_{0}(g)=n-1$ and $H_{n}(M, \mathbb{Z}) \neq 0$, then $M$ is isometric to $\mathbb{R} \times \Sigma$, with warped product metric $d t^{2}+\cosh ^{2}(t) h$, where $\Sigma$ is compact and $h$ is a metric on $\Sigma$ with Ric $\geq-(n-1)$.

Combined with a theorem of J. Lee [14] this generalizes a result of Witten-Yau [22] and Cai-Galloway [7]. The second part of the above theorem characterizes the borderline case completely. It is interesting to note that it is precisely the example given by Witten-Yau [22]. As a corollary of our theorem, we give a simple proof of a result concerning convex cocompact hyperbolic manifolds due to Bowen [6], Izeki [11] and Yue [23].

A Killing vector field on a conformally compact Einstein manifold $M$ extends to $\bar{M}$ and its restriction on the conformal infinity $\Sigma$ is a conformal vector field. In Section 4 the inverse problem is considered: given a conformal vector field on $\Sigma$ can it be extended to a Killing vector field on $M$ ? We show the answer is yes if we assume $M$ has non-positive curvature and there is an approximate Killing vector field.

A large class of conformally compact Einstein manifolds are given by convex cocompact hyperbolic manifolds. The conformal infinity of such manifolds is necessarily conformally flat. In Section 5 we study conformally compact Einstein

Received January 26, 2001. 
manifold whose conformal infinity is conformally flat. The main result in this section is

Theorem 1.2. Let $(M, g)$ be a conformally compact Einstein manifold whose conformal infinity is conformally flat and has nonnegative Yamabe invariant. If the sectional curvature satisfies $-\frac{3}{4}<K+1<\frac{3}{4}$, then $g$ is hyperbolic.

In the last section, several interesting examples of conformally compact Einstein manifolds are discussed. We also prove a non-existence result for certain spin manifolds.

Theorem 1.3. Let $M$ be the interior of a compact Spin manifold whose boundary is $T^{n}$ with its standard spin structure. Then there is no conformally compact Einstein metric on $M$ with the conformal infinity $\left(T^{n},[h]\right)$, where $h$ is a flat metric.

Compared with the known examples, this result illustrates the subtle role played by spin structures.

\section{Preliminaries}

Let $\bar{M}$ be a compact $(\mathrm{n}+1)$-dimensional manifold with boundary $\Sigma$. If $r$ is a smooth function on $\bar{M}$ with a first order zero on the boundary of $\bar{M}$, positive on $M$, then $r$ is called a defining function. A Riemannian metric $g$ on $M=\operatorname{Int} \bar{M}$ is called conformally compact if for any defining function $r, \bar{g}=r^{2} g$ extends as a smooth metric on $\bar{M}$. The restriction of $\bar{g}$ to $\Sigma$ gives a metric on $\Sigma$. This metric changes by a conformal factor if the defining function is changed, so $\Sigma$ has a well-defined conformal structure $c$. We call $(\Sigma, c)$ the conformal infinity of $(M, g)$. If $g$ satisfies the Einstein equation $\operatorname{Ric}(g)+n g=0$ we say $(M, g)$ is a conformally compact Einstein manifold.

The following lemma is very useful.

Lemma 2.1 (see [8]). A metric $h \in c$ on $\Sigma$ determines a unique defining function $r$ in a collar neighborhood of $\Sigma$ such that

$$
g=r^{-2}\left(d r^{2}+h_{r}\right),
$$

where $h_{r}$ is an $r$-dependent family of metrics on $\Sigma$ with $\left.h_{r}\right|_{r=0}=h$.

By the Einstein equation the expansion of $h_{r}$ is of the following form [9]. For $n$ odd,

$$
h_{r}=h_{(0)}+h_{(2)} r^{2}+(\text { even powers })+h_{(n-1)} r^{n-1}+h_{(n)} r^{n}+\ldots,
$$

where the $h_{(j)}$ are tensors on $\Sigma$, and $h_{(n)}$ is trace-free with respect to $h$. The tensors $h_{(j)}$ for $j \leq n-1$ are locally formally determined by the metric $h$, but $h_{(n)}$ is formally undetermined.

For $n$ even the analogous expansion is

$$
h_{r}=h_{(0)}+h_{(2)} r^{2}+(\text { even powers })+k r^{n} \log r+h_{(n)} r^{n}+\ldots,
$$

where the $h_{(j)}$ are locally determined for $j \leq n-2, k$ is locally determined and trace-free, but $h_{(n)}$ is formally undetermined. 
Conformally compact Einstein manifolds were first studied by Graham and Lee [8] and they proved the following local existence theorem.

Theorem 2.1. Let $M=B^{n+1}$ be the ball and $h_{0}$ the standard metric on the sphere $S^{n}$. For any metric $h$ on $S^{n}$ which is sufficiently close to $h_{0}$ in $C^{2, \alpha}$ norm if $n \leq 4$, or $C^{3, \alpha}$ norm if $n=3$, for some $0<\alpha<1$, then there exists a smooth conformally compact Einstein metric $g$ on $M$ with conformal infinity $\left(S^{n}, h\right)$.

During the last couple of years such manifolds have appeared in string theory as the mathematical framework for the AdS/CFT correspondence which gives a close connection between conformal field theory and supergravity. Therefore the study of conformally compact Einstein manifolds has become even important for physics. Inspired by physical considerations concerning the AdS/CFT correspondence Witten-Yau [22] proved the following theorem for the positive Yamabe invariant case in 1999. Shortly after Cai-Galloway [7] established the zero Yamabe invariant case.

Theorem 2.2. Let $(M, g)$ be a conformally compact Einstein manifold. If its conformal infinity $(\Sigma,[h])$ has non-negative Yamabe invariant, then

$$
H_{n}(M, \mathbb{Z})=0 \text {. }
$$

In particular, $\Sigma$ is connected.

For more background information we refer the reader to Graham-Lee [8], Lee [14], Graham-Witten [9] and Witten-Yau [22].

\section{A homology vanishing theorem}

We first state a general technical lemma.

Lemma 3.1. Let $\theta$ be a harmonic 1-form on a Riemannian manifold $M$ of dimension $n+1$. Then

$$
|\nabla \theta|^{2} \geq \frac{n+1}{n}|\nabla| \theta||^{2}
$$

Proof. We introduce a 2-tensor $H$ by

$$
H(u, v)=\nabla_{u} \theta(v) .
$$

Since $\theta$ is harmonic, $H$ is symmetric and has trace zero. Given $p \in M$ we choose a local orthonormal frame $\left\{e_{0}, e_{1}, \ldots, e_{n}\right\}$ such that at $p$

$$
\theta\left(e_{0}\right)=|\theta|, \theta\left(e_{i}\right)=0, \text { for } i=1, \ldots, n .
$$

In the following we do the calculation at $p$.

$$
\begin{aligned}
|\nabla \theta|^{2}-\left.|\nabla| \theta\right|^{2} & =\sum_{i, j=0}^{n} H\left(e_{i}, e_{j}\right)^{2}-\sum_{j=0}^{n} \frac{\left\langle\nabla_{e_{j}} \theta, \theta\right\rangle^{2}}{|\theta|^{2}} \\
& =\sum_{i, j=0}^{n} H\left(e_{i}, e_{j}\right)^{2}-\sum_{j=0}^{n} H\left(e_{0}, e_{j}\right)^{2}
\end{aligned}
$$




$$
\begin{aligned}
& =\sum_{i \neq 0} H\left(e_{i}, e_{j}\right)^{2} \\
& \geq \sum_{i=1}^{n} H\left(e_{i}, e_{0}\right)^{2}+\sum_{i=1}^{n} H\left(e_{i}, e_{i}\right)^{2} \\
& \geq \sum_{i=1}^{n} H\left(e_{i}, e_{0}\right)^{2}+\frac{1}{n} H\left(e_{0}, e_{0}\right)^{2} \\
& \geq \frac{1}{n}|\nabla| \theta||^{2} .
\end{aligned}
$$

i. e. $|\nabla \theta|^{2} \geq \frac{n+1}{n}|\nabla| \theta||^{2}$.

If equality holds at $p$ then $H$ has an eigenvalue $\mu$ of multiplicity $n$ and another eigenvalue $-n \mu$ such that

$$
H\left(e_{i}, e_{j}\right)=\mu \delta_{i j}, H\left(e_{0}, e_{0}\right)=-n \mu, H\left(e_{0}, e_{i}\right)=0
$$

for $i, j=1, \ldots, n$.

For a complete, non-compact Riemannian manifold $(M, g)$, we denote the infimum of the $L^{2}$ spectrum of its Laplacian $-\triangle$ by $\lambda_{0}(g)$. It can be defined as

$$
\lambda_{0}(g)=\inf _{f \in C_{c}^{1}(M)} \frac{\int_{M}|\nabla f|^{2}}{\int_{M} f^{2}} .
$$

Let $\mathcal{H}^{k}(M)$ be the space of $L^{2}$ harmonic $k$-forms.

Theorem 3.1. Let $(M, g)$ be a conformally compact Riemannian manifold of dimension $n+1$ with Ric $\geq-n$.

1. If $\lambda_{0}(g)>n-1$, then $\mathcal{H}^{1}(M)=0$.

2. If $\lambda_{0}(g)=n-1$ and $\mathcal{H}^{1}(M) \neq 0$, then $M$ is isometric to $\mathbb{R} \times \Sigma$, with warped product metric $d t^{2}+\cosh ^{2}(t) h$, where $\Sigma$ is compact and $h$ is a metric on $\Sigma$ with Ric $\geq-(n-1)$.

Proof. Suppose that $\theta$ is a non-zero $L^{2}$ harmonic 1-form on $M$. By the Bochner formula we have

$$
\frac{1}{2} \triangle|\theta|^{2}=|\nabla \theta|^{2}+\operatorname{Ric}(\theta, \theta) \geq|\nabla \theta|^{2}-n|\theta|^{2} .
$$

By Lemma 3.1

$$
\frac{1}{2} \triangle|\theta|^{2} \geq\left.\frac{n+1}{n}|\nabla| \theta\right|^{2}-n|\theta|^{2} .
$$

By Mazzeo's work (see [15] and [16]) we have $f \triangleq|\theta|=O\left(r^{n}\right)$ (Mazzeo proved an asymptotic expansion for $\theta$ by constructing a parametrix for the Hodge Laplacian in a collar neighborhood of the conformal infinity. His method originally gives the decay rate $|\theta|=O\left(r^{n-1}\right)$. By using the well-known fact that an $L^{2}$ harmonic form on a complete Riemannian manifold is both closed and coclosed, one can 
improve the decay rate to get $|\theta|=O\left(r^{n}\right)$. See [21] for more details). This implies that $\phi \triangleq f^{(n-1) / n}=O\left(r^{n-1}\right) \in L^{2}(M)$. From (3.1) we get

$$
\frac{n}{2(n-1)} \operatorname{div} \nabla \phi^{2} \geq \frac{n}{n-1}|\nabla \phi|^{2}-n \phi^{2} .
$$

Therefore

$$
\int_{M^{\delta}}|\nabla \phi|^{2}-(n-1) \phi^{2} \leq \int_{\Sigma^{\delta}} \phi \frac{\partial \phi}{\partial \nu}
$$

where $\nu$ is the outer unit normal of $\Sigma^{\delta}$. By geometric-mean inequality

$$
\left|\int_{\Sigma^{\delta}} \phi \frac{\partial \phi}{\partial \nu}\right| \leq \int_{\Sigma^{\delta}} \phi|\nabla \phi| \leq \frac{1}{2} \int_{\Sigma^{\delta}}\left(\phi^{2}+|\nabla \phi|^{2}\right) .
$$

As $\int_{M} \phi^{2}+|\nabla \phi|^{2}<\infty$, there exists a sequence $\delta_{k} \rightarrow 0$ such that $\int_{\Sigma^{\delta_{k}}} \phi^{2}+$ $|\nabla \phi|^{2} \rightarrow 0$. Taking limit in (3.2), we get

$$
\int_{M}|\nabla \phi|^{2} \leq(n-1) \int_{M} \phi^{2}
$$

This implies that $\lambda_{0} \leq n-1$. Thus $\mathcal{H}^{1}(M)=0$ if $\lambda_{0}(g)>n-1$.

If $\lambda_{0}(g)=n-1$ and there is a nonzero harmonic form $\theta$, then all the inequalities in the above argument are equalities. First we have

$$
-\triangle \phi=(n-1) \phi \text {. }
$$

By Harnack inequality $\phi$ is positive everywhere. We also have

$$
|\nabla \theta|^{2}=\frac{n+1}{n}|\nabla| \theta||^{2} .
$$

Let $X$ be the unit vector field dual to the 1 -form $\theta /|\theta|$. By the proof of Lemma 3.1 , we have

$$
\begin{gathered}
\nabla_{X} \theta(X)=-n \mu \\
\nabla_{u} \theta(v)=\mu\langle u, v\rangle \\
\nabla_{X} \theta(u)=0
\end{gathered}
$$

for $u, v$ orthogonal to $X$. Then

$$
\nabla_{u} \theta(v)=u f\langle X, v\rangle+f\left\langle\nabla_{u} X, v\right\rangle
$$

By (3.4)(3.5) and (3.6) we get

$$
\begin{gathered}
\nabla_{X} X=0 \\
X f=-n \mu \\
\left\langle\nabla_{u} X, v\right\rangle=\frac{\mu}{f}\langle u, v\rangle, \\
u f=0,
\end{gathered}
$$

for $u, v$ orthogonal to $X$. It follows that $\nabla f=-n \mu X$. Let $\gamma: \mathbb{R} \rightarrow M$ be any trajectory of $X$, which is a geodesic by (3.7). We have

$$
\frac{d f \circ \gamma}{d t}=-n \mu \circ \gamma
$$


We choose a local orthonormal frame $\left\{e_{0}, e_{1}, \ldots, e_{n}\right\}$ near $\gamma(t)$ such that $e_{0}=X$. By (3.7) (3.9) and (3.10) we calculate

$$
\begin{aligned}
\triangle f(\gamma(t)) & =X X f+\sum_{i=1}^{n}\left(e_{i} e_{i} f-\nabla_{e_{i}} e_{i} f\right) \\
& =\frac{d^{2} f}{d t^{2}}-\sum_{i=1}^{n}\left\langle\nabla f, \nabla_{e_{i}} e_{i}\right\rangle \\
& =\frac{d^{2} f}{d t^{2}}+n \mu \sum_{i=1}^{n}\left\langle X, \nabla_{e_{i}} e_{i}\right\rangle \\
& =\frac{d^{2} f}{d t^{2}}-n \mu \sum_{i=1}^{n}\left\langle\nabla_{e_{i}} X, e_{i}\right\rangle \\
& =\frac{d^{2} f}{d t^{2}}-n^{2} \mu^{2} / f .
\end{aligned}
$$

By (3.3) and the fact that $\phi=f^{n /(n-1)}$ we obtain

$$
\triangle f=|\nabla f|^{2} / n f-n f=n \mu^{2} / f-n f .
$$

By (3.11)(3.12) and (3.13) we get the following ODE

$$
\frac{d^{2} f \circ \gamma}{d t^{2}}=\frac{n+1}{n f \circ \gamma}\left(\frac{d f \circ \gamma}{d t}\right)^{2}-n f \circ \gamma
$$

This ODE can be easily solved to give

$$
f \circ \gamma(t)=a \cosh ^{-n}(t+c),
$$

where $a>0$ and $c$ are constants. By (3.8)

$$
\mu \circ \gamma(t)=-\frac{1}{n} \frac{d}{d t} f \circ \gamma(t)=a \cosh ^{-n-1}(t+c) \sinh (t+c) .
$$

Let $B=\max f$. If $\gamma(0) \in \Sigma \triangleq\{x \in M \mid f(x)=B\}$, then $c=0$ and $a=B$. Therefore the flow of $X$ moves any level set of $f$ to another level set. It is now clear that $\Sigma$ is a compact hypersurface and the flow of $X$ contracts $M$ to $\Sigma$. Therefore $M=\mathbb{R} \times \Sigma$ and the metric $g$ must be the form

$$
g=d t^{2}+h_{i j}(t, x) d x^{i} d x^{j},
$$

where $x$ is coordinates on $\Sigma$. By (3.9) (3.14) and (3.15) we have

$$
\frac{1}{2} \frac{\partial h_{i j}}{\partial t}=\left\langle\nabla_{\frac{\partial}{\partial x_{i}}} \frac{\partial}{\partial t}, \frac{\partial}{\partial x_{j}}\right\rangle=\frac{\mu}{f} h_{i j}=\frac{\sinh (t)}{\cosh (t)} h_{i j} .
$$

This implies that

$$
h_{i j}(t, x)=\cosh ^{2}(t) h_{i j}(0, x) .
$$

Therefore $g=d t^{2}+\cosh ^{2}(t) h$ where $h$ is a metric on $\Sigma$. As Ric $g \geq-n$, it is easy to see that $\operatorname{Ric}_{h} \geq-(n-1)$. 
Conversely for such a manifold $M$ one can easily verify that $\cosh ^{-n} d t$ is an $L^{2}$ harmonic 1-form and

$$
-\triangle \cosh ^{-(n-1)}(t)=(n-1) \cosh ^{-(n-1)}(t) .
$$

Hence $\lambda_{0}=n-1$ and $\mathcal{H}^{1}(M) \neq 0$.

Corollary 3.1. Let $(M, g)$ be a conformally compact Riemannian manifold with Ric $\geq-n$ and $\lambda_{0}(g)>n-1$, then $H_{n}(M, \mathbb{Z})=0$. In particular, the conformal infinity is connected.

Proof. It has been shown by R. Mazzeo [15] that $\mathcal{H}^{1}(M)$ is isomorphic to $H^{1}(\bar{M}, \Sigma)$. Therefore $H^{1}(\bar{M}, \Sigma)=0$ by Theorem 3.1. It then follows $H^{1}(\bar{M}, \Sigma ; \mathbb{Z})=0$ for we know it is also torsion free. By Lefshetz duality $H_{n}(\bar{M}, \mathbb{Z})=0$. To prove the second claim we use the cohomology exact sequence

$$
0 \rightarrow H^{0}(\bar{M}) \rightarrow H^{0}(\Sigma) \rightarrow H^{1}(\bar{M}, \Sigma) \rightarrow \cdots .
$$

As $H^{1}(\bar{M}, \Sigma)=0, H^{0}(\bar{M}) \rightarrow H^{0}(\Sigma)$ is an isomorphism. Therefore $\Sigma$ is connected.

The significance of $H_{n}(M, \mathbb{Z})=0$ for AdS/CFT correspondence is that it rules out the existence of wormholes (see [22] for a detailed explanation). About $\lambda_{0}(g)$, the following result is known.

Theorem 3.2 (J. Lee [14]). Let $(M, g)$ be a conformally compact Einstein manifold. If its conformal infinity has positive Yamabe invariant, then $\lambda_{0}(g)=n^{2} / 4$.

Combining the previous two theorems we get

Corollary 3.2. Let $M$ be the interior of a compact manifold whose boundary $\Sigma$ has more than one components. Let $c$ be a conformal structure on $\Sigma$ such that each component has nonnegative Yamabe invariant. Then there is no conformal compact Einstein metric with $(\Sigma, c)$ as its conformal infinity.

Remark. In the Corollary we have to assume that each component of the conformal infinity has positive Yamabe invariant. Actually the result is established if one of the components has positive Yamabe invariant (Witten-Yau [22]) or zero Yamabe invariant (Cai-Galloway [7]).

Remark. In another sense, Theorem 3.1 is obviously stronger than the result of Witten-Yau and Cai-Galloway who prove $H_{n}(M, \mathbb{Z})=0$ under the condition that the conformal infinity has nonnegative Yamabe invariant. Theorem 3.1 shows that this is true even for certain manifolds whose conformal infinity has negative Yamabe invariant. Consider a hyperbolic manifold $M=B^{n+1} / \Gamma$ where $\Gamma$ is a torsion-free convex cocompact discrete subgroup of Isom ${ }^{+}\left(\mathbb{H}^{n+1}\right)$. Such $M$ is a conformally compact Einstein (hyperbolic) manifold with conformally infinity $\left(S^{n}-\Lambda(\Gamma)\right) / \Gamma$ where $\Lambda(\Gamma)$ is the limit set of $\Gamma$. For such manifolds 
Sullivan [18] proved that $\lambda_{0}<n^{2} / 4$ iff $\Lambda(\Gamma)$ has Hausdorff dimension $\delta>n / 2$, and in that case $\lambda_{0}=\delta(n-\delta)$. By Theorem 3.1 we get

$$
\text { if } \delta<n-1 \text { then } H_{n}(M, \mathbb{Z})=0 \text {. }
$$

If $\delta>(n-2) / 2$ by a result due to Schoen-Yau [17] the conformal infinity actually has negative Yamabe invariant.

In 1979, Bowen [6] proved that the Hausdorff dimension of the limit set of a quasi-Fuchsian group acting on $S^{2}$, which is not Fuchsian, is greater than 1 . It had been conjectured that the same result should be true in higher dimensions. The conjecture was solved by Izeki [11] and Yue [23], independently using quite different methods. As an application of Theorem 3.1, we can give a simple proof of a special case of their theorem.

Corollary 3.3. Let $M^{n+1}=\mathbb{H}^{n+1} / \Gamma$ be an oriented convex compact hyperbolic manifold whose limit set $\Lambda(\Gamma)$ is a round sphere $S^{n-1} \subset S^{n}$. Suppose that $\Gamma^{\prime}$ is another convex cocompact Kleinian group which is isomorphic to $\Gamma$ as an abstract group. Then the Hausdorff dimension $\delta\left(\Gamma^{\prime}\right) \geq n-1$. Moreover, if $\delta\left(\Gamma^{\prime}\right)=n-1$, $\Gamma^{\prime}$ is conjugate to $\Gamma$ by a Möbius transformation.

Proof. Since $\Lambda(\Gamma)$ is a round sphere $S^{n-1} \subset S^{n}$, the convex hull of $\Lambda(\Gamma)$ is $B^{n} \subset B^{n+1}$ and $\Sigma=B^{n} / \Gamma$ is a compact hyperbolic manifold. $M$ is the warped product $\mathbb{R} \times \Sigma$, hence $H_{n}(M, \mathbb{R})=\mathbb{R}$. The convex cocompact hyperbolic manifold $M^{\prime}=\mathbb{H}^{n+1} / \Gamma^{\prime}$ is homotopy equivalent to $M$ because both are $K(\Gamma, 1)$ manifolds. Therefore $H_{n}\left(M^{\prime}, \mathbb{R}\right)=\mathbb{R}$. By Theorem 3.1, $\lambda_{0} \leq n-1$. Thus by Sullivan's theorem

$$
\delta\left(\Gamma^{\prime}\right)>n / 2 \quad \text { and } \quad \delta\left(\Gamma^{\prime}\right)\left(n-\delta\left(\Gamma^{\prime}\right)\right) \leq n-1 .
$$

It follows that $\delta\left(\Gamma^{\prime}\right) \geq n-1$.

If $\delta\left(\Gamma^{\prime}\right)=n-1$, by Theorem $3.1 M^{\prime}$ is the warped product of $\mathbb{R}$ with a compact hyperbolic manifold $\Sigma^{\prime}$ whose fundamental group is $\Gamma^{\prime}$. Since $\Gamma^{\prime}$ is isomorphic to $\Gamma$ as an abstract group, $\Sigma^{\prime}$ is isometric to $\Sigma$ by Mostow rigidity. Therefore $M^{\prime}$ is isometric to $M$, or equivalently, $\Gamma^{\prime}$ is conjugate to $\Gamma$ by a Möbius transformation.

\section{Asymptotic symmetry}

Let $(M, g)$ be a conformally compact Einstein manifold with conformal infinity $(\Sigma,[h])$. By Lemma 2.1 we can write the metric near infinity as

$$
g=r^{-2}\left(d r^{2}+h_{i j}(r, x) d x^{i} d x^{j}\right),
$$

where $\left(x^{1}, \ldots, x^{n}\right)$ are local coordinates on $\Sigma$. Let $X=a(r, x) \frac{\partial}{\partial r}+b^{i}(r, x) \frac{\partial}{\partial x^{i}}$ be a vector field on $M$. By calculation we have the following formulas

$$
L_{X} g\left(\frac{\partial}{\partial r}, \frac{\partial}{\partial r}\right)=2 r^{-2}\left(\frac{\partial a}{\partial r}-a / r\right)
$$


$L_{X} g\left(\frac{\partial}{\partial x^{i}}, \frac{\partial}{\partial x^{j}}\right)=\left(\frac{\partial b^{k}}{\partial x^{i}}+b^{l} \Gamma_{i l}^{k}\right) \frac{h_{k j}}{r^{2}}+\left(\frac{\partial b^{k}}{\partial x^{j}}+b^{l} \Gamma_{j l}^{k}\right) \frac{h_{k i}}{r^{2}}-2 \frac{a}{r^{3}} h_{i j}+\frac{a}{r^{2}} \frac{\partial h_{i j}}{\partial r}$, where $\Gamma_{i j}^{k}$ are Christoffel symbols of the metric $h_{i j}(r, x) d x^{i} d x^{j}$.

Proposition 4.1. Let $X$ be a Killing vector field. Then $X$ extends to a smooth vector field on $\bar{M}$ whose restriction on $\Sigma$ is a conformal vector field.

Proof. Write $X=a(r, x) \frac{\partial}{\partial r}+b^{i}(r, x) \frac{\partial}{\partial x^{i}}$ near infinity. As $X$ is a Killing vector field we have $L_{X} g=0$. By (4.1) we get

$$
\frac{\partial a}{\partial r}-a / r=0
$$

It follows $a(r, x)=r a_{0}(x)$. By (4.2) we get

$$
\frac{\partial b^{j}}{\partial r} h_{i j}+\frac{\partial a}{\partial x^{i}}=0 .
$$

It implies $b^{j}(r, x)=b^{j}\left(r_{0}, x\right)-\int \frac{\partial a}{\partial x^{i}} h^{i j}(r, x) d r$. Hence $X$ extends to a vector field on $\bar{M}$ and its restriction on $\Sigma$ is $Y=b^{i}(0, x) \frac{\partial}{\partial x^{i}}$. By (4.3) we have

$$
\left(\frac{\partial b^{k}}{\partial x^{i}}+b^{l} \Gamma_{i l}^{k}\right) h_{k j}+\left(\frac{\partial b^{k}}{\partial x^{j}}+b^{l} \Gamma_{j l}^{k}\right) h_{k i}-2 \frac{a}{r} h_{i j}+a \frac{\partial h_{i j}}{\partial r}=0 .
$$

When we retric to the boundary $r=0$ we get

$$
\left(\frac{\partial b^{k}}{\partial x^{i}}+b^{l} \Gamma_{i l}^{k}\right) h_{k j}+\left(\frac{\partial b^{k}}{\partial x^{j}}+b^{l} \Gamma_{j l}^{k}\right) h_{k i}=2 a_{0}(x) h_{i j},
$$

where we use the fact that $\left.\frac{\partial h_{i j}}{\partial r}\right|_{r=0}=0$. Note (4.4) is equivalent to $L_{Y} h=2 a_{0} h$ and thus $Y$ is a conformal vector field on $\Sigma$.

Lemma 4.1. Let $(M, g)$ be an $n+1$-dimensional Riemannian manifold with $\operatorname{Ric}(g)=-n g$. A vector field $X$ on $M$ satisfies

$$
\frac{1}{2} \triangle L_{X} g=\frac{1}{2} L_{\triangle X-n X} g-\stackrel{\circ}{R} L_{X} g
$$

where, in terms of an orthonormal frame, $(\stackrel{\circ}{R} h)_{i j}=R_{i k j l} h_{k l}$ for a symmetric 2-tensor $h$.

Proof. Let $F_{t}$ be the (local) flow generated by $X$. Let $g_{t}=F_{t}^{*} g$. We have $-n g_{t}=\operatorname{Ric}\left(g_{t}\right)$. We differentiate both sides with respect to $t$ and use the formula (1.180a) in Besse [5]

$$
\begin{aligned}
-n L_{X} g & =\left.\frac{d}{d t} \operatorname{Ric}\left(g_{t}\right)\right|_{t=0} \\
& =-\frac{1}{2} \triangle L_{X} g-\stackrel{\circ}{R} L_{X} g-\delta^{*} \delta L_{X} g-D^{2}(\operatorname{div} X)+\operatorname{Ric} \circ L_{X} g .
\end{aligned}
$$


As Ric $\circ L_{X} g=-n L_{X} g$, we get

$$
-\frac{1}{2} \triangle L_{X} g-\stackrel{\circ}{R} L_{X} g-\delta^{*} \delta L_{X} g-D^{2}(\operatorname{div} X)=0 .
$$

If we identify a vector field with its dual 1-form we have the following identity

$$
\delta L_{X} g=\triangle X+\nabla \operatorname{div} X+\operatorname{Ric}\left(e_{j}, X\right) e_{j}=\triangle X+\nabla \operatorname{div} X-n X .
$$

It is also easy to verify

$$
\delta^{*} \nabla \operatorname{div} X=-D^{2}(\operatorname{div} X) .
$$

By (4.5) (4.6) and (4.7) we get

$$
-\frac{1}{2} \triangle L_{X} g-\stackrel{\circ}{R} L_{X} g+\frac{1}{2} L_{\triangle X-n X} g=0 .
$$

This is the formula we want.

Definition 4.1. A vector field $X$ on $\bar{M}$ is asymptotic Killing if $L_{X} g=O\left(r^{n-2}\right)$.

Theorem 4.1. Let $(M, g)$ be a conformally compact Einstein manifold with nonpositive sectional curvature. For any asymptotic Killing vector field $Y$ on $M$ there is a Killing vector field $X$ such that $\left.X\right|_{\Sigma}=\left.Y\right|_{\Sigma}$.

Proof. First by general elliptic theory on conformally compact manifolds e. g. , see [14], we can solve

$$
\triangle Z-n Z=-(\triangle Y-n Y)
$$

to get a vector field $Z$ which satisfies $Z=O\left(r^{n-2}\right)$. Let $X=Y+Z$. Then $X$ is asymptotic Killing and $\triangle X-n X=0$. By Lemma 4.1 we have

$$
\frac{1}{2} \triangle\left|L_{X} g\right|^{2}=\left|\nabla L_{X} g\right|^{2}-2\left\langle\stackrel{\circ}{R} L_{X} g, L_{X} g\right\rangle .
$$

As $\left|L_{X} g\right|^{2}=O\left(r^{2 n}\right)$, integration by parts gives

$$
\int_{M}\left|\nabla L_{X} g\right|^{2}=2 \int_{M}\left\langle\stackrel{\circ}{R} L_{X} g, L_{X} g\right\rangle .
$$

On the other hand we have the following formula from Besse [5]

$$
(\delta d+d \delta) L_{X} g=-\triangle L_{X} g-\stackrel{\circ}{R} L_{X} g-n L_{X} g .
$$

By integration by parts we get

$$
\int_{M}\left|\nabla L_{X} g\right|^{2} \geq \int_{M}\left\langle\stackrel{\circ}{R} L_{X} g, L_{X} g\right\rangle+n\left|L_{X} g\right|^{2} .
$$

By (4.8) and (4.9) we get

$$
\int_{M}\left\langle\stackrel{\circ}{R} L_{X} g, L_{X} g\right\rangle \geq n \int_{M}\left|L_{X} g\right|^{2} .
$$


As $M$ has nonpositive sectional curvature, by Theorem 12. 71 in Besse [5] we have

$$
\left\langle\stackrel{\circ}{R} L_{X} g, L_{X} g\right\rangle \leq n\left|L_{X} g\right|^{2}
$$

Combining with (4.10) we conclude $L_{X} g=0$, i. e. $X$ is a Killing vector field.

Given a conformal vector field on $\Sigma$, one may try to construct an asymptotic Killing vector field. In general this seems to be difficult to do. Nevertheless we have the following

Theorem 4.2. Let $\left(M_{1}, g_{1}\right)$ and $\left(M_{2}, g_{2}\right)$ be two conformally compact Einstein manifolds with the same conformal infinity $(\Sigma,[h])$. If $Y$ is a Killing vector field on $M_{1}$, then there is an almost Killing vector field $X$ on $M_{2}$ such that $\left.X\right|_{\Sigma}=\left.Y\right|_{\Sigma}$.

Proof. Identifying $M_{1}$ and $M_{2}$ near the infinity we can write $g_{1}$ and $g_{2}$ as

$$
\begin{aligned}
& g_{1}=r^{-2}\left(d r^{2}+\tilde{h}_{r}\right), \\
& g_{1}=r^{-2}\left(d r^{2}+h_{r}\right) .
\end{aligned}
$$

By the discussion at the end of Section 2 we have

$$
h_{r}=\tilde{h}_{r}+O\left(r^{n}\right)
$$

Let $Y=a(r, x) \partial / \partial r+b^{i}(r, x) \partial / \partial x^{i}$. Extending $Y$ to the compact part of $M_{2}$ we get a vector field $X$ on $M_{2}$. By (4.1) (4.2) (4.3) and (4.14) we get

$$
L_{X} g_{2}=L_{Y} g_{2}=L_{Y} g_{1}+O\left(r^{n-2}\right)=O\left(r^{n-2}\right) .
$$

i. e. $X$ is an almost Killing vector field.

Remark. If we further assume $\left(M_{2}, g_{2}\right)$ is nonpositively curved in the above theorem, we can produce a Killing vector field on $M_{2}$ by Theorem 4.1. It is possible to use this result to prove global uniqueness of nonpositively curved Einstein metrics with certain conformal infinities.

\section{Conformal infinity which is conformally flat}

Let $(M, g)$ be a Riemannian manifold of dimension $n+1$. We denote its Weyl tensor by $W$. We will first prove a Bochner formula for $W$ by straightforward calculation.

Lemma 5.1. Let $X, Y$ and $Z$ be vector fields on $M$ and $\left\{e_{\alpha}\right\}$ a local orthonormal frame. Then

$$
\nabla_{X} W\left(e_{\alpha}, Y, e_{\alpha}, Z\right)=0
$$


Proof. It is obvious that the left hand side is independent of the orthonormal frame, hence we need only to check it pointwise using a normal frame. Then we have

$$
\begin{aligned}
& \nabla_{X} W\left(e_{\alpha}, Y, e_{\alpha}, Z\right) \\
= & X\left(W\left(e_{\alpha}, Y, e_{\alpha}, Z\right)\right)-W\left(e_{\alpha}, \nabla_{X} Y, e_{\alpha}, Z\right)-W\left(e_{\alpha}, Y, e_{\alpha}, \nabla_{X} Z\right) \\
= & 0
\end{aligned}
$$

where in the last step we simply use the fact that the trace of $W$ is zero.

If $(M, g)$ is Einstein, we have

$$
R=\frac{s}{2 n(n+1)} g \circ g+W .
$$

It follows that $W$ satisfies the second Bianchi identity.

Lemma 5.2. Let $(M, g)$ be an Einstein manifold of dimension $n+1$. Then

$$
\frac{1}{2} \triangle|W|^{2}=|\nabla W|^{2}+K,
$$

where $K=-2 n|W|^{2}-2 R_{i \alpha j \beta} W_{\alpha \beta k l} W_{i j k l}-4 R_{i \alpha k \beta} W_{j \alpha l \beta} W_{i j k l}$.

Proof. For any $p \in M$ we choose a local orthonormal frame $\left\{e_{i}\right\}$ which is normal at $p$. The following calculation is done at $p$. As usual we sum over repeated indices. By Lemma 5.1 and the first and second Bianchi identities we have

$$
\begin{aligned}
& \nabla_{e_{\alpha}} \nabla_{e_{\alpha}} W\left(e_{i}, e_{j}, e_{k}, e_{l}\right) \\
& =e_{\alpha}\left(\nabla_{e_{\alpha}} W\left(e_{i}, e_{j}, e_{k}, e_{l}\right)\right) \\
& =-e_{\alpha}\left(\nabla_{e_{i}} W\left(e_{j}, e_{\alpha}, e_{k}, e_{l}\right)+\nabla_{e_{j}} W\left(e_{\alpha}, e_{i}, e_{k}, e_{l}\right)\right) \\
& =-e_{\alpha}\left[e_{i} W_{j \alpha k l}+e_{j} W_{\alpha i k l}-W\left(\nabla_{e_{i}} e_{j}, e_{\alpha}, e_{k}, e_{l}\right)-W\left(e_{j}, \nabla_{e_{i}} e_{\alpha}, e_{k}, e_{l}\right)\right. \\
& \left.-W\left(e_{j}, e_{\alpha}, \nabla_{e_{i}} e_{k}, e_{l}\right)-W\left(e_{j}, e_{\alpha}, e_{k}, \nabla_{e_{i}} e_{l}\right)\right]-W\left(\nabla_{e_{j}} e_{\alpha}, e_{i}, e_{k}, e_{l}\right) \\
& \left.-W\left(e_{\alpha}, \nabla_{e_{j}} e_{i}, e_{k}, e_{l}\right)-W\left(e_{\alpha}, e_{i}, \nabla_{e_{j}} e_{k}, e_{l}\right)-W\left(e_{\alpha}, e_{i}, e_{k}, \nabla_{e_{j}} e_{l}\right)\right] \\
& =-e_{i} e_{\alpha} W_{j \alpha k l}-e_{j} e_{\alpha} W_{\alpha i k l}+W\left(\nabla_{e_{\alpha}} \nabla_{e_{i}} e_{j}, e_{\alpha}, e_{k}, e_{l}\right) \\
& +W\left(e_{j}, \nabla_{e_{\alpha}} \nabla_{e_{i}} e_{\alpha}, e_{k}, e_{l}\right)+W\left(e_{j}, e_{\alpha}, \nabla_{e_{\alpha}} \nabla_{e_{i}} e_{k}, e_{l}\right) \\
& \left.+W\left(e_{j}, e_{\alpha}, e_{k}, \nabla_{e_{\alpha}} \nabla_{e_{i}} e_{l}\right)\right]+W\left(\nabla_{e_{\alpha}} \nabla_{e_{j}} e_{\alpha}, e_{i}, e_{k}, e_{l}\right) \\
& +W\left(e_{\alpha}, \nabla_{e_{\alpha}} \nabla_{e_{j}} e_{i}, e_{k}, e_{l}\right)+W\left(e_{\alpha}, e_{i}, \nabla_{e_{\alpha}} \nabla_{e_{j}} e_{k}, e_{l}\right) \\
& \left.+W\left(e_{\alpha}, e_{i}, e_{k}, \nabla_{e_{\alpha}} \nabla_{e_{j}} e_{l}\right)\right] \\
& =-e_{i}\left[\nabla_{e_{\alpha}} W\left(e_{j}, e_{\alpha}, e_{k}, e_{l}\right)+W\left(\nabla_{e_{\alpha}} e_{j}, e_{\alpha}, e_{k}, e_{l}\right)\right. \\
& +W\left(e_{j}, \nabla_{e_{\alpha}} e_{\alpha}, e_{k}, e_{l}\right)+W\left(e_{j}, e_{\alpha}, \nabla_{e_{\alpha}} e_{k}, e_{l}\right) \\
& \left.+W\left(e_{j}, e_{\alpha}, e_{k}, \nabla_{e_{\alpha}} e_{l}\right)\right]-e_{j}\left[\nabla_{e_{\alpha}} W\left(e_{\alpha}, e_{i}, e_{k}, e_{l}\right)\right. \\
& +W\left(\nabla_{e_{\alpha}} e_{\alpha}, e_{i}, e_{k}, e_{l}\right)+W\left(e_{\alpha}, \nabla_{e_{\alpha}} e_{i}, e_{k}, e_{l}\right) \\
& \left.+W\left(e_{\alpha}, e_{i}, \nabla_{e_{\alpha}} e_{k}, e_{l}\right)+W\left(e_{\alpha}, e_{i}, e_{k}, \nabla_{e_{\alpha}} e_{l}\right)\right] \\
& +W\left(\nabla_{e_{\alpha}} \nabla_{e_{i}} e_{j}, e_{\alpha}, e_{k}, e_{l}\right)+W\left(e_{j}, \nabla_{e_{\alpha}} \nabla_{e_{i}} e_{\alpha}, e_{k}, e_{l}\right) \\
& \left.+W\left(e_{j}, e_{\alpha}, \nabla_{e_{\alpha}} \nabla_{e_{i}} e_{k}, e_{l}\right)+W\left(e_{j}, e_{\alpha}, e_{k}, \nabla_{e_{\alpha}} \nabla_{e_{i}} e_{l}\right)\right]
\end{aligned}
$$




$$
\begin{aligned}
& +W\left(\nabla_{e_{\alpha}} \nabla_{e_{j}} e_{\alpha}, e_{i}, e_{k}, e_{l}\right)+W\left(e_{\alpha}, \nabla_{e_{\alpha}} \nabla_{e_{j}} e_{i}, e_{k}, e_{l}\right) \\
& \left.+W\left(e_{\alpha}, e_{i}, \nabla_{e_{\alpha}} \nabla_{e_{j}} e_{k}, e_{l}\right)+W\left(e_{\alpha}, e_{i}, e_{k}, \nabla_{e_{\alpha}} \nabla_{e_{j}} e_{l}\right)\right] \\
= & e_{i}\left[\nabla_{e_{k}} W\left(e_{j}, e_{\alpha}, e_{l}, e_{\alpha}\right)+\nabla_{e_{l}} W\left(e_{j}, e_{\alpha}, e_{\alpha}, e_{k}\right)\right] \\
& +e_{j}\left[\nabla_{e_{k}} W\left(e_{\alpha}, e_{i}, e_{l}, e_{\alpha}\right)+\nabla_{e_{l}} W\left(e_{\alpha}, e_{i}, e_{\alpha}, e_{k}\right)\right] \\
& +W\left(R_{i \alpha} e_{j}, e_{\alpha}, e_{k}, e_{l}\right)+W\left(e_{j}, R_{i \alpha} e_{\alpha}, e_{k}, e_{l}\right) \\
& +W\left(e_{j}, e_{\alpha}, R_{i \alpha} e_{k}, e_{l}\right)+W\left(e_{j}, e_{\alpha}, e_{k}, R_{i \alpha} e_{l}\right) \\
& +W\left(R_{j \alpha} e_{\alpha}, e_{i}, e_{k}, e_{l}\right)+W\left(e_{\alpha}, R_{j \alpha} e_{i}, e_{k}, e_{l}\right) \\
& +W\left(e_{\alpha}, e_{i}, R_{j \alpha} e_{k}, e_{l}\right)+W\left(e_{\alpha}, e_{i}, e_{k}, R_{j \alpha} e_{l}\right) \\
= & W\left(R_{i \alpha} e_{j}, e_{\alpha}, e_{k}, e_{l}\right)+W\left(e_{j}, R_{i \alpha} e_{\alpha}, e_{k}, e_{l}\right) \\
& +W\left(e_{j}, e_{\alpha}, R_{i \alpha} e_{k}, e_{l}\right)+W\left(e_{j}, e_{\alpha}, e_{k}, R_{i \alpha} e_{l}\right) \\
& +W\left(R_{j \alpha} e_{\alpha}, e_{i}, e_{k}, e_{l}\right)+W\left(e_{\alpha}, R_{j \alpha} e_{i}, e_{k}, e_{l}\right) \\
& +W\left(e_{\alpha}, e_{i}, R_{j \alpha} e_{k}, e_{l}\right)+W\left(e_{\alpha}, e_{i}, e_{k}, R_{j \alpha} e_{l}\right) \\
= & -2 n W_{i j k l}+W\left(R_{i \alpha} e_{j}, e_{\alpha}, e_{k}, e_{l}\right)+W\left(e_{j}, e_{\alpha}, R_{i \alpha} e_{k}, e_{l}\right) \\
& +W\left(e_{j}, e_{\alpha}, e_{k}, R_{i \alpha} e_{l}\right)+W\left(e_{\alpha}, R_{j \alpha} e_{i}, e_{k}, e_{l}\right) \\
& +W\left(e_{\alpha}, e_{i}, R_{j \alpha} e_{k}, e_{l}\right)+W\left(e_{\alpha}, e_{i}, e_{k}, R_{j \alpha} e_{l}\right)
\end{aligned}
$$

This implies

$$
\begin{aligned}
\frac{1}{2} \triangle|W|^{2}= & |\nabla W|^{2}+\langle\triangle W, W\rangle \\
= & |\nabla W|^{2}-2 n|W|^{2}+2 R_{i \alpha j \beta} W_{\beta \alpha k l} W_{i j k l} \\
& +2 R_{i \alpha k \beta} W_{j \alpha \beta l} W_{i j k l}+2 R_{i \alpha l \beta} W_{j \alpha k \beta} W_{i j k l} \\
= & |\nabla W|^{2}-2 n|W|^{2}-2 R_{i \alpha j \beta} W_{\alpha \beta k l} W_{i j k l} \\
& -4 R_{i \alpha k \beta} W_{j \alpha l \beta} W_{i j k l} .
\end{aligned}
$$

Thus we get the formula

$$
\frac{1}{2} \triangle|W|^{2}=|\nabla W|^{2}+K
$$

Following [19], for fixed k, j, i,h we introduce a local skew symmetric tensor $u_{l m}^{(k j i h)}$ by

$$
\begin{aligned}
u_{l m}^{(k j i h)}= & W_{l j i h} \delta_{m k}+W_{k l i h} \delta_{m j}+W_{k j l h} \delta_{m i}+W_{k j i l} \delta_{m h} \\
& -W_{m j i h} \delta_{l k}-W_{k m i h} \delta_{l j}-W_{k j m h} \delta_{l i}-W_{k j i m} \delta_{l h} .
\end{aligned}
$$

The following two lemmas are stated in [19] (our notation is slightly different). The proof is a long but simple calculation.

\section{Lemma 5.3.}

$$
R_{l m p q} u_{l m}^{(k j i h)} u_{p q}^{(k j i h)}=8 K .
$$




\section{Lemma 5.4.}

$$
\sum_{\substack{l, m \\ k, j, i, h}} u_{l m}^{(k j i h)} u_{l m}^{(k j i h)}=8 n|W|^{2} .
$$

In dimension three, there is the following beautiful construction due to Bers [4]. Let $\Sigma$ be a compact oriented surfaces of genus $\geq 2$. Given two conformal structures $c_{1}$ and $c_{2}$ on $\Sigma$, there is a conformally compact hyperbolic metric on $\Sigma \times(0,1)$ with the conformal infinity $\left(\Sigma, c_{1}\right) \sqcup\left(\Sigma, c_{2}\right)$. In higher dimensions, we can not expect such a picture. Let $\Gamma$ be a Kleinian group acting on $S^{n}$. We denote its limit set by $\Lambda(\Gamma)$. Suppose that $S^{n}-\Lambda(\Gamma)$ has a component $\Omega$ on which $\Gamma$ acts freely. Then $\Omega / \Gamma$ is a manifold with a conformally flat structure.

Proposition 5.1. If $\Omega / \Gamma$ is a compact manifold with a hyperbolic metric in its conformal structure, then $\Gamma$ is a Fuchsian group. Hence $\Lambda(\Gamma)$ is round $(n-1)$ dimensional sphere in $S^{n}$.

Proof. Let $h_{0}$ be the standard metric on $S^{n}$. By the assumption there is a complete hyperbolic metric $h=\rho^{-2} h_{0}$ on $\Omega$, where $\rho$ is a positive function. By the formula relating the Ricci tensor of $h$ and that of $h_{0}$ we get

$$
D^{2} \rho=\frac{\triangle \rho}{n} h_{0},
$$

where $D^{2}$ and $\triangle$ are taken with respect to $h_{0}$. The above formula simply means that $\nabla \rho$ is a conformal vector field of $h_{0}$. Then $\exists \xi \in S^{n}$ and a constant $c$ such that $\rho(x)=x \cdot \xi+c$. For $h$ to be complete $\Omega$ must be one of the sphere caps bounded by the $n$-1-dimensional round sphere on which $\rho$ is zero. Therefore $\Gamma$ fixes a round $S^{n-1}$ in $S^{n}$. This implies that $\Gamma$ is a Fuchsian group.

Let $\left(M, g_{0}\right)$ be a compact hyperbolic manifold of dimension at least three with another conformally flat structure $c$, e. g. If $M$ contains a totally geodesic hypersurface, the bending construction produces exotic conformally flat structures. By the proposition there exists no conformally compact hyperbolic metric on $M \times(0,1)$ with the conformal infinity $\left(M,\left[g_{0}\right]\right) \sqcup(M, c)$. But it is possible to have a conformally compact Einstein metric.

Theorem 5.1. Let $(M, g)$ be a conformally compact Einstein manifold whose conformal infinity is conformally flat and has nonnegative Yamabe invariant. If the sectional curvature satisfies $-\frac{3}{4}<K+1<\frac{3}{4}$, then $g$ is hyperbolic.

Proof. First we show that the Weyl tensor $W$ decays rapidly. As the conformal infinity is conformally flat we can take a metric $h$ in the conformal class which is Euclidean on a local chart $U \subset \Sigma$. On $(0, \epsilon) \times U$ the metric $\bar{g}=r^{-2}\left(d r^{2}+h\right)$ is hyperbolic and thus has zero Weyl tensor. As $g=r^{-2}\left(d r^{2}+h_{r}\right)=\bar{g}+O\left(r^{n-2}\right)$ on $(0, \epsilon) \times U$ we have $W=O\left(r^{n-2}\right)$. Therefore we get

$$
|W|^{2}=O\left(r^{2 n+4}\right) \text {. }
$$


By (5.1), Lemma 5.3 and 5.4 we have

$$
\triangle|W|^{2} \geq|\nabla W|^{2}-n\|\mathcal{R}\||W|^{2}
$$

where $\|\mathcal{R}\|$ stands for the norm of the curvature operator on 2 -forms. By the Berger inequality [12] we have the following estimate under our pinching assumption

$$
\|\mathcal{R}\|<\frac{3}{2}
$$

Therefore

$$
\triangle|W|^{2} \geq|\nabla W|^{2}-\frac{n^{2}}{4}|W|^{2}
$$

This implies, in view of $(5.2)$

$$
\int|\nabla W|^{2} \leq \frac{n^{2}}{4} \int|W|^{2}
$$

As $\lambda_{0}=n^{2} / 4$, we conclude $W \equiv 0$. Therefore $g$ is hyperbolic.

\section{Examples and a non-existence result}

Let $\left(N, g_{0}\right)$ be a Riemannian manifold of dimension $n+1-k$ such that Ric $=-(n-k)$. Consider the following metric on $B^{k} \times N$

$$
g=\frac{4}{\left(1-|x|^{2}\right)^{2}}\left(d x^{2}+\frac{\left(1+|x|^{2}\right)^{2}}{4} g_{0}\right)
$$

where $x$ is the coordinates on $B^{k}$. Then $g$ is a conformally compact Einstein metric. The conformal infinity is the $S^{k-1} \times N$ with the product metric. If we use polar coordinates on the hyperbolic space the metric can be written in the following form

$$
g=d t^{2}+\sinh ^{2}(t) d \omega^{2}+\cosh ^{2}(t) g_{0}
$$

where $d \omega^{2}$ is the standard metric on $S^{k-1}$.

Remark. If $\left(N, g_{0}\right)$ is hyperbolic, then $g$ is also hyperbolic. We write $N=$ $B^{n+1-k} / \Gamma$, where $\Gamma$ is a torsion-free cocompact lattice in $O(1, n+1-k)$. Then $B^{k} \times N=B^{n+1} / \Gamma$, here we view $\Gamma$ as a discrete subgroup in $O(1, n+1)$ through the inclusion $O(1, n+1-k) \rightarrow O(1, n+1)$.

Remark. The referee pointed out that the above metric is the Euclidean version of a well-known static solution to the vacuum Einstein equations (referred to as the Kottler $\mathrm{k}=0$ solution).

When $k=2$, the above Einstein metric was constructed in Bérard Bergery [4]. He also constructed Einstein metrics with negative scalar curvature on $B^{2} \times N$, where $N$ is a compact Einstein manifold of positive or zero scalar curvature. We show his metrics, properly normalized, are conformally compact. 
We take a slightly different perspective as we encountered first their Lorentzian counterparts in general relativity.

Consider the ADS-Schwarzschild spacetime in general relativity

$$
g=-f(r) d t^{2}+f(r)^{-1} d r^{2}+r^{2} d \omega^{2},
$$

where $d \omega^{2}$ is the standard metric on $S^{n-1}$ and $f(r)=1+r^{2}-\frac{M}{r^{n-2}}$ with $M>0$ a constant. The metric is defined on $\mathbb{R} \times\left(r_{0}, \infty\right) \times S^{n-1}$, where $r_{0}>0$ is the zero of $f$, and satisfies the Einstein equation $\operatorname{Ric}(g)=-n g$. If we replace $t$ by it we get a Riemannian Einstein metric

$$
g=f(r)^{-1} d r^{2}+f(r) d u^{2}+r^{2} g_{0}
$$

on $\left(r_{0}, \infty\right) \times S^{1} \times M$, where $u$ is periodic of length $L$ and $\left(N, g_{0}\right)$ is a compact (n-1)-dimensional manifold such that $\operatorname{Ric}\left(g_{0}\right)=(n-2) g_{0}$. That we can replace $\left(S^{n-1}, d \omega^{2}\right)$ by $\left(N, g_{0}\right)$ is because the Einstein equation Ric $(g)=-n g$ only involves the Ricci curvature of $g_{0}$. The metric $g$ is not complete when $r$ goes to $r_{0}$. However we will show that this is caused by the failure of the coordinates we use, something also happened to the Schwarzschild spacetime in general relativity. We change coordinates by solving the following ODE

$$
\left\{\begin{array}{l}
r^{\prime \prime}(t)=r+\frac{n-2}{2} \frac{M}{r^{n-1}}, \\
r(0)=r_{0}, r^{\prime}(0)=0 .
\end{array}\right.
$$

This easily implies that $r^{\prime}(t)=\sqrt{f(r)}$ and thus the metric takes the form

$$
g=d t^{2}+h(t) d u^{2}+r(t)^{2} g_{0}
$$

on $(0, \infty) \times S^{1} \times N$, where $h(t)=f \circ r(t)$. From the ODE we see that $r$ is analytic and even in $t$. Hence $h(t)$ is also even in $t$. Note $h(0)=0$ and $h^{\prime \prime}(0)=2\left(r_{0}+\frac{n-2}{2} M / r_{0}^{n-1}\right)^{2}$. If we chose $L$ such that $L h^{\prime \prime}(0) / 2=2 \pi$ then $g$ can be extended to $t=0$ and we get a complete metric on $\mathbb{R}^{2} \times N$. From its original form this metric is obviously conformally compact.

If $M=0$ the metric becomes

$$
g=\left(1+r^{2}\right)^{-1} d r^{2}+\left(1+r^{2}\right) d u^{2}+r^{2} g_{0}
$$

initially defined on $(0, \infty) \times S^{1} \times N$. Let $r=\sinh (t)$ we get

$$
g=d t^{2}+\cosh ^{2}(t) d u^{2}+\sinh ^{2}(t) g_{0} .
$$

We can not extend the metric as before. However if $g_{0}$ is the standard metric on $S^{n-1}$, the part $d t^{2}+\sinh ^{2}(t) g_{0}$ is the hyperbolic metric on $\mathbb{H}^{n}$ in polar coordinates. Therefore $g$ is a complete metric on $\mathbb{H}^{n} \times S^{1}$. In fact $g$ is hyperbolic. It can be obtained as $\mathbb{H}^{n+1} / \Gamma$ where $\Gamma$ is the elementary group generated by a dilation. This is a conformally compact hyperbolic metric on $\mathbb{H}^{n} \times S^{1}$ with conformal infinity $S^{n-1} \times S^{1}$.

By the same argument, given a compact $(n-1)$-dimensional Ricci-flat manifold $\left(N, g_{0}\right)$ we can show that

$$
g=\left(r^{2}-M / r^{n-2}\right)^{-1} d r^{2}+\left(r^{2}-M / r^{n-2}\right) d u^{2}+r^{2} g_{0}, r>M^{1 / n}
$$


is conformally compact Einstein metric on $B^{2} \times N$, where $u$ has period $4 \pi / n M^{1 / n}$ (to resolve the conical singularity at $r=M^{1 / n}$ ). The conformal infinity is easily seen to be $\left(S^{1} \times N,\left[d \theta^{2}+h\right]\right)$. In the following we specialize to the special case that $\left(N, g_{0}\right)$ is the torus $T^{n-1}$ with a flat metric. Let $\tau_{0}$ be the spin structure on the torus defined by the trivial spin bundle. Note $B^{2} \times T^{n-1}$ is a spin manifold, but none of its spin structures induces $\tau_{0}$ on its boundary $T^{n}$. As the spin cobordism group in dimension 3 is trivial [13], there is a compact spin 4manifold $\Sigma^{4}$ with boundary $\left(T^{3}, \tau_{0}\right)$. Therefore $\Sigma^{4} \times T^{n-3}$ is a spin manifold with boundary $\left(T^{n}, \tau_{0}\right)$. We have shown on $B^{2} \times T^{n-1}$ there is a conformally compact Einstein metric whose conformal infinity is $T^{n}$ with a flat metric, however this is not true for $\Sigma^{4} \times T^{n-3}$ by the following theorem.

Theorem 6.1. Let $M$ be the interior of a compact Spin manifold with boundary $\left(T^{n}, \tau_{0}\right)$. Then there is no conformally compact Einstein metric on $M$ with the conformal infinity $\left(T^{n},[h]\right)$, where $h$ is a flat metric.

Proof. The proof is based on the idea due to Andersson and Dahl [1]. Suppose there is such a metric $g$. Then near infinity we can choose a defining function $r$ such that $g=r^{-2}\left(d r^{2}+h_{r}\right)$, with $h_{0}$ a flat metric on $T^{n}$. The Einstein condition implies that

$$
\begin{gathered}
h_{r}=h_{0}+O\left(r^{n}\right), \\
\operatorname{tr}_{h_{0}} h_{r}=n+O\left(r^{2 n}\right) .
\end{gathered}
$$

Consider the hyperbolic manifold $(0, \infty) \times T^{n}, g=t^{-2}\left(d t^{2}+h_{0}\right)$ with the spin structure defined by the trivial $\operatorname{Spin}(n+1)$-bundle. It has Killing spinors $\psi=t^{-1 / 2}\left(1-\sqrt{-1} e_{1} \cdot\right) u$ (with respect to the obvious trivialization of the tangent bundle), where $u$ is a spinor vector and $e_{1}=-t \frac{\partial}{\partial t}$. We use $\psi$ to construct an asymptotically Killing spinor on $M$. This is possible for the induced spin structures on $T^{n}$ are the same. By (6.2) and (6.3), the same calculation as in [1] shows that the mass of this asymptotic Killing spinor is zero. Therefore $M$ has a Killing spinor. We refer to [1] for detail. Then by a theorem of Baum [2] $M$ is a warped product $\mathbb{R} \times T^{n}$ with metric $g=d t^{2}+e^{2 t} h_{0}$. But then it has two ends and one of them $(t \rightarrow-\infty)$ is a cusp. This contradiction shows that such a metric $g$ can not exist.

\section{Acknowledgment}

I wish to thank my advisor, Prof. Rick Schoen, for guiding my study and sharing his ideas and insights with me over the last three years. Without his guidance, encouragement and valuable suggestions, this work would not be possible. Thanks are also due to Prof. Rafe Mazzeo for his interests in my work and for many helpful discussions. He explained to me how to resolve the conical singularity for the metrics discussed in the last section. 


\section{References}

[1] L. Andersson and M. Dahl, Scalar curvature rigidity for asymptotically locally hyperbolic manifolds, Ann. Global Anal. Geom. 16 (1998), no. 1, 1-27.

[2] H. Baum, Complete Riemannian manifolds with imaginary Killing spinors, Ann. Global Anal. Geom. 7 (1989), no. 3, 205-226.

[3] L. Bérard Bergery, Sur de Nouvelles Variétés Riemanniannes d'Einstein, Publications de l'Institut E. Cartan (1982), no. 4, 1-60.

[4] L. Bers, Simultaneous uniformization, Bull. Amer. Math. Soc. 66 (1960), 94-97.

[5] A. Besse, Einstein Manifolds, Springer-Verlag, Berlin, 1987.

[6] R. Bowen, Hausdorff dimension of quasi-circles, Inst. Hautes tudes Sci. Publ. Math. No. 50, (1979), 11-25.

[7] M. Cai and G. J. Galloway, Boundaries of zero scalar curvature in the ADS/CFT Correspondence, preprint hep-th/0003046.

[8] R. Graham and J. Lee, Einstein metrics with prescribed conformal infinity on the ball, Advances in Math. 87 (1991), 186-225.

[9] R. Graham and E. Witten, Conformally anomaly of submanifold observables in ADS/CFT Correspondence, preprint hep-th/9901021.

[10] M. Henningson and K. Skenderis, Holograph and the Weyl anomaly, preprint hepth/9812032.

[11] H. Izeki, Limit sets of Kleinian groups and conformally flat Riemannian manifolds, Invent. Math. 122 (1995), no. 3, 603-625.

[12] H. Karcher, A short proof of Berger's curvature tensor estimates, Proc. Amer. Math. Soc. 26(1970) 642-644.

[13] H. B. Lawson and M.-L. Michelson, Spin Geometry, Princeton Univ. Press, 1989.

[14] J. Lee, The spectrum of an asymptotically hyperbolic Einstein manifold, Comm. Anal. Geom. 3 (1995), no. 1-2, 253-271.

[15] R. Mazzeo, The Hodge cohomology of a conformally compact metric, J. Differential Geom. 28 (1988), no. 2, 309-339.

[16] R. Mazzeo and R. Phillips, Hodge theory on hyperbolic manifolds, Duke Math. J. 60 (1990), no. 2, 509-559.

[17] R. Schoen and S.-T. Yau, Conformally flat manifolds, Kleinian groups and scalar curvature, Invent. Math. 92 (1988), no. 1, 47-71.

[18] D. Sullivan, Related aspects of positivity in Riemannian geometry, J. Differential Geom. 25 (1987), no. 3, 327-351.

[19] S. Tachibana, A theorem of Riemannian manifolds of positive curvature operator, Proc. Japan Acad. 50 (1974), 301-302.

[20] Xiaodong Wang, A new proof of Lee's Theorem on the spectrum of conformally compact Einstein manifolds, to appear in Comm. Anal. Geom.

[21] Xiaodong Wang, On the $L^{2}$ cohomology of convex cocompact hyperbolic manifolds, to appear in Duke Mathematical Journal.

[22] E. Witten and S.-T. Yau, Connectedness of the boundary in the ADS/CFT Correspondence, preprint hep-th/9910245.

[23] C. Yue, Dimension and rigidity of quasi-Fuchsian representations, Ann. of Math. (2) 143 (1996), no. 2, 331-355.

Department of Mathematics, Mit, Cambridge, MA 02139.

E-mail address: xwang@math.mit.edu 